\title{
Un développement rapide de la formation technique et professionnelle
}

\section{Lisa Bydanova et Ganat Dalabayev}

\section{OpenEdition}

1 Journals

Édition électronique

URL : https://journals.openedition.org/ries/2648

DOI : $10.4000 /$ ries.2648

ISSN : 2261-4265

Éditeur

France Education international

\section{Édition imprimée}

Date de publication : 1 décembre 2012

Pagination : 17-18

ISSN : 1254-4590

\section{Référence électronique}

Lisa Bydanova et Ganat Dalabayev, « Un développement rapide de la formation technique et professionnelle », Revue internationale d'éducation de Sèvres [En ligne], 61 | décembre 2012, mis en ligne le 06 février 2015, consulté le 06 juillet 2021. URL : http://journals.openedition.org/ries/2648 ; DOI : https://doi.org/10.4000/ries.2648

Ce document a été généré automatiquement le 6 juillet 2021.

(c) Tous droits réservés 


\title{
Un développement rapide de la formation technique et professionnelle
}

\author{
Lisa Bydanova et Ganat Dalabayev
}

1 La rénovation de la formation technique et professionnelle (FTP) est un enjeu très important aujourd'hui au Kazakhstan. Ce nouveau pays $^{1}$, en pleine croissance économique, cherche à renforcer ce secteur pour pérenniser son développement, basé jusqu'à présent sur l'exportation des ressources naturelles².

2 Au cours des cinq dernières années, la FTP a connu une importante progression quantitative, avec des financements étatiques conséquents. Le financement public du secteur a été multiplie par 3,1 sur cette période. Les dépenses en travaux de rénovation ont augmenté de 1,3 fois entre 2008 et 2010. Leur montant total représentait en 2010 3,3 milliards de tenge, soit environ 16 millions d'euros. Le nombre d'établissements dans ce secteur a augmenté de $14 \%$ et le nombre d'étudiants de $20 \%$, passant de 499900 en 2005 à 603700 en 2010. À titre de comparaison, le nombre d'étudiants inscrits dans les programmes de l'enseignement supérieur était de 620442 en 2010.

3 Cette forte expansion du secteur fait qu'aujourd'hui, le nombre d'étudiants en formation professionnelle sur dix mille habitants est de loin supérieur à ceux parmi les plus élevés des pays de l'OCDE. Cet indicateur représente 368 au Kazakhstan, alors qu'il s'élève à 180 en France, à 171 en Allemagne ou à 135 au Royaume-Uni ${ }^{4}$.

4 Par ailleurs, ce secteur a fait objet de nombreuses reformes structurelles. Une importante réforme, qui consiste à unifier les établissements de formation technique et professionnelle sur l'ensemble du pays pour leur donner un statut unique, est en cours de réalisation. Elle obligera certains lycées professionnels à changer de statut pour devenir des «colleges» pouvant dispenser ainsi des programmes de formation professionnelle de différents niveaux au sein d'un même établissement (technicien de base, technicien de niveau intermédiaire ou supérieur). En outre, le ministère souhaite remédier au manque de passerelles et à la faible articulation entre les parcours de formations. À ce titre, il a mis en place deux parcours de formation innovants dits 
«parcours intégrés»: il s'agit d'alléger les programmes de formation de niveau supérieur en prenant en compte les acquis d'apprentissage validés dans les programmes de formation antérieurs; ces parcours pilotes concernent les domaines de l'extraction pétrolière.

5 Un travail important a également été mené pour rénover les contenus de formation. Entre 2008 et 2010, 158 maquettes de formation ont été révisées par le ministère avec l'appui des employeurs et des experts internationaux. En 2010, 710 plans de cours ont été revus et 65 nouveaux cours ont été créés.

Cependant, malgré cette forte expansion quantitative et des efforts de modernisation, la qualité des formations dispensées n'est pas toujours à la hauteur des efforts consentis. Certains problèmes pourraient mettre en péril les réformes lancées : la faible attractivité du métier d'enseignant due à de mauvaises conditions de travail et de salaire (comme dans l'enseignement général), un pilotage d'établissements inefficace, un manque d'équipements et d'infrastructures pédagogiques et de mauvaises conditions d'accueil des étudiants.

7 En outre, les formations professionnelles et techniques sont souvent perçues comme une «voie de garage» empruntée par ceux qui n'ont pas un niveau académique suffisant ou qui manquent de ressources pour financer leurs études universitaires. Ces dernières, malgré leur coût (variant de 300 à 2000 dollars par an), et quelle que soit leur qualité académique ou professionnelle, bénéficient d'un grand prestige au sein de la population, les filières de l'économie et de droit restant les plus prisées, alors que les formations en sciences techniques ou d'ingénieurs tendent à être désertées par les étudiants.

L'autre difficulté importante que rencontre le secteur de la formation professionnelle (comme celui de l'enseignement supérieur en général) est le décalage entre le contenu des formations et les besoins du marché du travail. D'une part, ce dernier est essentiellement composé de petites entreprises peu organisées entre elles (seulement $5 \%$ des entreprises, par exemple, font partie d'un syndicat) et d'autre part, les établissements de formation sont peu incités à promouvoir ce rapprochement, car leurs ressources dépendent peu de ce critère. En effet, si les établissements de formation sont tenus de fournir des statistiques sur l'insertion de leurs diplômés, la plupart se contentent de déclarer un taux avoisinant $100 \%$, assez éloigné de la réalité.

\section{NOTES}

1. Voir Lisa Bydanova et Gulbarshin Ospanova, "Le système éducatif du Kazakhstan ", Revue internationale d'éducation de Sèvres, $\mathrm{n}^{\circ}$ 59, 2012, 19-25.

2. $55 \%$ du budget de l'État proviennent des exportations pétrolières; $16 \%$ du PIB du pays proviennent des exportations des ressources métallurgiques (manganèse, fer, chrome, charbon et potassium).

3. Ce nombre est passé de 787 en 2005 à 894 en 2010.

4. Source : Rapport national sur l'état du système éducatif au Kazakhstan, p. 40. 


\section{INDEX}

Keywords : vocational education, technical education, upper secondary vocational schools, upper secondary technical schools, educational system

Mots-clés : enseignement professionnel, enseignement technique, lycée professionnel, lycée technique, système éducatif

Index géographique : Kazakhstan

Palabras claves : enseñanza profesional, enseñanza técnica, escuela secundaria profesional, escuela secundaria técnica, sistema educativo

\section{AUTEURS}

\section{LISA BYDANOVA}

Chargée de programmes au Centre international d'études pédagogiques, Département coopération en éducation, et chercheur associé de l'IREDU, Dijon (France)

\section{GANAT DALABAYEV}

Directeur-adjoint du Centre national du développement de la formation professionnelle au Kazakhstan, où il supervise l'élaboration d'un programme pilote de formation dans le domaine de l'extraction pétrolière. Diplômé de l'Université publique d'agriculture de Moscou, il a occupé plusieurs postes à responsabilité en matière de formation professionnelle au Kazakhstan 